\title{
COMPETÊNCIAS INDIVIDUAIS VOLTADAS À SUSTENTABILIDADE NAS ORGANIZAÇÕES
}

\author{
Günther Pires Weissböck ${ }^{1}$ \\ Silvio Roberto Stefano ${ }^{2}$
}

\begin{abstract}
Resumo: $O$ estudo teve por objetivo principal realizar uma análise referente às produções científicas na base de periódicos da CAPES que contenham em seu título os termos "organizational sustainability" e ou "individual competences" no período de 2006 a 2015, caracterizando-se como uma pesquisa exploratória e descritiva. Foram analisados 79 artigos, identificando as variáveis: ano, idioma original, revista, palavras-chave, número de autores por artigo, quantidade de publicação por autor, vínculo institucional e país de vinculação. Os resultados apontaram que os artigos são predominantemente escritos em inglês, por mais de um autor e na sua maioria originários dos Estados Unidos da América.
\end{abstract}

Palavras-Chave: Competências; Sustentabilidade; Gestão Ambiental e Social.

\section{INDIVIDUAL COMPETENCES FOCUSED ON ORGANIZATIONAL SUSTAINABILITY}

\begin{abstract}
The study had the main objective conduct an analysis regarding the scientific production based on the CAPES periodic that contains in your title the terms "Organizational Sustainability" and "Individual Competences" in the period 2006-2015, featuring as a exploratory and descriptive research. We analyzed 79 articles, identifying variables: year, original language, magazines, keywords, number of authors per article, institutional link and linking country. The results show that the articles are predominantly if written in English, by more than one author and its originating from United States of America.
\end{abstract}

Keywords: Competences; Sustainability; Environmental and Social Management.

\section{INTRODUÇÃO}

A sustentabilidade organizacional é um tema que vem ganhando relevância nos últimos trinta anos, se tornando um fator de competitividade nas empresas, sendo um grande objeto de estudo no meio educacional e empresarial. 
Desta maneira, Osorio et al. (2005) citam a importância dos valores organizacionais e individuais, explicando que a sustentabilidade organizacional se estabelece em três dimensões: econômica, ambiental e social. A dimensão econômica se relaciona com o quão bem a empresa está com o fator financeiro, além de serviços e produtos com qualidade. Já o fator ambiental se relaciona com os aspectos da geração atual em garantir a satisfação das próximas gerações, e a dimensão social se refere ao fator de bem estar com os indivíduos da organização, concluindo que para que haja um ambiente sustentável, as ações devem ser voltadas para essas três dimensões.

Hoff, Barin-Cruz \& Pedrozo (2009) complementam que as organizações devem se adequar às tendências e exigências do mundo moderno, e é necessário considerar a construção da sustentabilidade como um processo, e deve ocorrer de forma gradativa para que se possa adaptar as características do paradigma centrado na sustentabilidade à realidade organizacional.

Diante disso, relacionado ao conceito de competências, Resende (2000) entende-se que esse conceito é uma transformação de conhecimento e habilidades para atingir um objetivo ou resultado. Diante disso, competência é um conjunto de conhecimentos que englobam a formação, treinamento, experiência juntamente com o comportamento dos interesse do indivíduo.

As competências organizacionais e individuais tem que caminhar juntas dentro da organização, a fim de se complementarem tornando o âmbito organizacional mais competitivo, além de contribuir e repassar o conhecimento para todos os indivíduos presentes dentro deste ambiente.

Desta forma, as organizações cada vez mais reconhecem a importância das práticas de competências aplicadas a sustentabilidade no mundo dos negócios, pois essa prática sustentável vem ganhando com o passar do tempo, onde as organizações devem ser competitivas e efetivas de forma sustentável.

Frente a isso, nota-se a grande crescente dos trabalhos científicos e acadêmicos voltados para as competências individuais e sustentabilidade organizacional, compreendendo o campo de estudo por meio de pesquisas bibliométricas e verificações quantitativas.

Partindo dessa proposição, o estudo tem por objetivo principal analisar a produção contida na base de periódicos da CAPES que contém em seu título os termos "individual competences" e "organizational sustainability" no período de 2006 à 2015, e visa identificar quanto aos artigos: o ano, idioma original, revista publicada e palavra-chave, e identificar quanto aos autores: número de autores por artigo, quantidade de publicações por autor, 


\section{COMPETÊNCIAS INDIVIDUAIS VOLTADAS À SUSTENTABILIDADE NAS ORGANIZAÇÕES}

vínculo institucional e país de vinculação. Por fim, verificar a característica da produção científica em torno das palavras-chave através dos dados coletados. Esse artigo está estruturado em referencial teórico, metodologia, análises dos resultados e considerações finais.

\section{REFERENCIAL TEÓRICO}

Partindo da premissa de que esta pesquisa buscou fazer um levantamento das discussões teóricas e empírica sem artigos publicados acerca da sustentabilidade organizacional e das competências individuais no âmbito acadêmico, tanto a nível nacional quanto internacional, considera-se de suma importância discutir, mesmo que de forma breve, sobre o que se compreende sobre sustentabilidade e competências.

A sustentabilidade tem acarretado diversos debates no meio acadêmico e empresarial, em vista de que as questões sócio-ambientais se tornaram extremamente importantes no âmbito empresarial, já que muitas dessas empresas utilizam recursos naturais e do meio social para se manterem competitivas no mercado. Frente a isso, Santos et al. (2008) explica que os primeiros estudos sobre a sustentabilidade iniciaram-se nas ciências ambientais e ecológicas, trazendo à tona diversos complementos para as disciplinas de Econômica, Sociologia e Filosofia.

O termo sustentabilidade está relacionado com a necessidade de encontrar maneiras de interação da sociedade com a natureza, e o progresso é medido pela qualidade de vida ao invés de consumo material (Ferreira, 2005).

Desta maneira, Rosa (2007) afirma que a sustentabilidade é um movimento histórico que questiona a sociedade industrial enquanto modo de desenvolvimento e é considerada um conceito importado da ecologia, mas ainda deve ser provada nas sociedades humanas.

Dentro da sustentabilidade, no que diz respeito a sustentabilidade organizacional, Osorio et al. (2005) explicam que é apenas uma das diversas sustentabilidades que incrementam para o desenvolvimento sustentável, ou seja, quando ligada a diversos aspectos da sustentabilidade, conseguem alcançar um equilíbrio dos diversos sistemas sociais. $\mathrm{O}$ conjunto dessas características formam os pilares sociais, econômicos e ambiental para se alcançar o desenvolvimento sustentável.

Dyllick \& Hockerts (2002) complementam esse pensamento e afirmam que os pesquisadores da área de sustentabilidade devem desvendar como as organizações podem incrementar a sustentabilidade econômica, social e ambiental de uma forma que haja uma 37 Revista Competitividade e Sustentabilidade - ComSus, Paraná, v. 3, n. 2, p. 35-48, Jul /Dez. 2016. 
integração entre esses três pilares principais a fim de que seja a base para a clareza da sustentabilidade.

Buscando os desafios para alcançar essa base, Edwards (2009, p.5) afirma que:

Enfrentar o desafio da sustentabilidade envolve inerentemente transformação. As mudanças radicais necessárias para alguma forma sustentável da ecologia e da economia global irá envolver mudanças paradigmáticas não só nos mundos externos de produtos, comportamentos, tecnologias e estruturas económicas, mas também nos mundos internos de valores, propósitos e visões para o futuro. (tradução dos autores).

De acordo com Edwards (2009), o principal desafio para que se alcance essa base é a transformação das atividades externas e internas referentes aos pilares da sustentabilidade, buscando novos valores e propósitos com um pensamento futuro, levando em consideraçãoos aspectos de comportamento, dos produtos, a tecnologia e as estruturas econômicas no real momento em que se estabelece a empresa.

Ainda segundo o autor, deve-se compreender que as teorias de transformação para se obter a sustentabilidade organizacional é diversificada e oferecem diversas explicações de como as empresas podem se mover em relação aos princípios mais sustentáveis. Frente a isso, sabemos que cada empresa é diferente e mantêm seus propósitos, missões e valores, porém existem padrões de semelhança entre os indivíduos e grupos das empresas, fazendo com que as estruturas sociais que criam esses objetivos possam ser regularizados e descritos em termos com modelos baseados em estágio de desenvolvimento.

$\mathrm{Na}$ década de 1970, as empresas eram consideradas sustentáveis caso fossem economicamente saudáveis, ou seja, com um patrimônio e lucros em constante evolução, mesmo tendo dívidas. Com o passar do tempo, essas afirmativas foram se desdobrando até chegar a uma abordagem conhecida como o triple bottomline, que se remete aos resultados de uma organização não somente no aspecto de lucratividade mas também no desenvolvimento social e ambiental.

Frente a isso, segundo Isenmann et al.(2007), afirmam que esse conceito ganhou destaque no cenário devido ao fato de sinalizar as discussões sobre o tema, fazendo com que as organizações comunicassem para a sociedadeseus desempenhos sobre o seu aspecto econômico, social e ambiental.

De acordo com a Triple Bottom Line, Traci Douglas (2007,p.1) afirma que "[...] as a tool for identifying gaps between practice and promise, the TBL has spurred leaders and employees alike to find more ways to integrate sustainable practices into their day-to-day work." Frente a isso, entende-se que a Triple Bottom Line tem estimulado as organizações 


\section{COMPETÊNCIAS INDIVIDUAIS VOLTADAS À SUSTENTABILIDADE NAS ORGANIZAÇÕES}

para encontrar diversas maneiras de integrar as práticas sustentáveis no dia-a-dia da dinâmica das empresas.

E no que diz respeito ao conceito de competências, Resende (2000) afirma que competência e uma transformação de conhecimentos, habilidades e interesses em resultados reais, explicando que quando uma pessoa tem conhecimento e experiência e não sabe aplicar essas qualidades em favor de um objetivo, esse indivíduo não é competente. Partindo dessa afirmação, competência é um conjunto de conhecimentos que englobam a formação, treinamento, experiência juntamente com o comportamento relacionado com a vontade e interesse do indivíduo.

Fleury \& Fleury (2001) completam que o conceito de competências deve agregar valor econômico à organização e valor social ao indivíduo, explicando que existem duas dimensões de competência: as competências organizacionais e individuais. As competências organizacionais são definidas pelo conjunto de conhecimentos técnicos e habilidades que causam impacto em produtos nas organizações, fornecendo vantagem competitiva no mercado e valor percebido pelo cliente.Neste sentido, em âmbito organizacional pode ser associada aos elementos de estratégia competitiva das organizações que são a missão, visão e os valores.

Conforme Dutra (2008) a competência individual é uma ação da qual se juntam os conhecimentos, habilidades e atitudes pessoais e profissionais para alcançar um objetivo, em uma determinada situação, afirmando que os conhecimentos não adquirem status de competência a menos que sejam compartilhados e sua rede de conhecimento é fundamental para que a comunicação seja eficiente e possa gerar competência aos indivíduos. O termo competência pode ser associado à como saber agir, saber aprender, saber se engajar, assumir responsabilidade, dentre outros.

Partindo desta premissa, o mesmo autor entende que as competências devem ser observadas para que possam ser acompanhadas a fim de conceder um feedback ao indivíduo, e a quantidade de competências definidas para o acompanhamento não pode ser grande, pois ela dificulta esse processo, representando uma falta de estímulo aos responsáveis pelo feedback.

Diante deste aspecto, a competência individual é o resultado da troca de saberes e de interação entre atividades diferentes, a fim de atingir o desempenho esperado, sabendo alinhar competências individuais com as competências organizacionais. Assim Boucher (2007, p.1) explica que: 
Por muitos anos, o desempenho industrial tem sido considerado implicitamente como decorrente da otimização da tecnologia e de recursos (máquinas, estoques , etc.), tornada possível por organizações centralizadas. As exigências atuais do sistema industrial reintroduziram progressivamente a força de trabalho humana como a principal fonte de desempenho industrial. Fazendo este paradigma operacional requer a identificação e formalização cuidadosa da relação entre recursos humanos e desempenho industrial, através de conceitos como habilidades,competências ou know-how.(Tradução dos autores)

Desta forma, as organizações cada vez mais reconhecem a importância das práticas de competências aplicadas a sustentabilidade no mundo dos negócios, sendo cruciais para a concepção de abordagens adequadas para a realização de soluções sustentáveis. Logo, as competências se tornam um ativo estratégico para as organizações, onde buscam atingir um objetivo desejado de forma sustentável, se tornando um novo desafio.

Segundo Wieket. al. (2011) afirma que o tema de competências aplicada a sustentabilidade não são frequentemente usados ou discutidos na literatura, onde o campo de pesquisa ainda está em busca de conceitos mais abrangentes que se relacionam e integram as competências de sustentabilidade de uma forma significativa.

Ainda segundo o autor, para entendermos melhor esse termo, deve haver uma pesquisa sobre sustentabilidade e ligar os 5 fatores fundamentais de competências que são: as competências sobre sistema de pensamento, competência imaginária antecipada, competência normativa, competência estratégica e competência interpessoal.

Já Piccolotto et. al. (2010), complementa que os profissionais encontram-se em um cenário de mudanças econômicas, culturais e tecnológicas, e dessa forma é necessário usufruir de novas ferramentas para estar focado nas competências voltadas para a sustentabilidade.

Frente a isso, Stefano \& Alberton (2015), afirmam que a sustentabilidade traz um questionamento sobre as competências necessárias para que haja o seu desenvolvimento, podendo ser desenvolvida mediante as competências individuais de assumir responsabilidades, possuir iniciativa e como agir, lembrando que as competências voltadas a sustentabilidade devem agregar valor à organização, ao indivíduo, sociedade e também o meio ambiente.

Ainda segundo os mesmos autores, as competências individuais são um conjunto de saberes e entregas esperadas do indivíduo na função que ocupada dentro da organização de acordo com as estratégias organizacionais definidas, explicando as competências para a sustentabilidade com 5 principais características que são: Foco em pensamento sistêmico, preventiva, normativa, estratégica e interpessoal. 


\section{COMPETÊNCIAS INDIVIDUAIS VOLTADAS À SUSTENTABILIDADE NAS ORGANIZAÇÕES}

Essas características visam analisar coletivamente os sistemas complexos em diferentes segmentos e em diferentes escalas, dando um feedback sobre a situação, compreendendo e verificando sua estrutura, os principais componentes e dinâmicas, visando um cenário futuro relacionado com questões de sustentabilidade. Além disso, ter capacidade de especificar e aplicar valores de sustentabilidade, tendo uma compreensão profunda de conceitos estratégicos. Por fim, essas características tem por objetivo motivar e facilitar a colaboração de pesquisa sobre sustentabilidade participativa e resolução de problemas.

A lógica por trás dessa pesquisa é uma abordagem abrangente para a sustentabilidade e como resolver os problemas que requer a integração das cinco competências chave, e a partir disso obter resultados para a resolução de problemas integradas com a sustentabilidade.

\section{ASPECTOS METODOLÓGICOS}

Frente aos objetivos principais deste artigo, a metodologia presente se baseou na pesquisa exploratória e descritiva. Segundo Gil (2007) a pesquisa exploratória tem por finalidade ampliar o conhecimento a respeito de um determinado fenômeno, afirmando que esse tipo de pesquisa explora a realidade buscando aumentar o conhecimento para que se possa realizar uma pesquisa com um maior planejamento. Este planejamento é flexível, pois o pesquisador não possui clareza do problema nem da hipótese a serem investigados.

Ainda segundo o mesmo autor, a pesquisa descritiva tem por objetivo descrever com exatidão os fatos de uma determinada realidade, dando ênfase em coletar os dados e realizar uma análise, descrevendo as suas características.

O corpus documental foi constituído por artigos publicados na base de periódicos da CAPES que continham os termos "organizational sustainability' e "individual competences" em seu título, no período de 2006 a 2015. As categorias e variáveis que foram analisadas se basearam em:

1. Artigos: ano, idioma original, revista e palavra-chave.

2. Autores dos artigos: número de autores presentes por artigo, quantidade de publicações por cada autor, vínculo institucional e o país vinculado.

Foi realizada a leitura individual dos artigos estudados para identificar cada variável com o auxilio da planilha Excel ${ }^{\circledR}$ para contabilizá-las. Por fim, foi utilizado a pesquisa descritiva para analisar as informações dos 79 artigos encontrados. 


\section{ANÁLISE DE DADOS}

A seguir apresenta-se 5 tabelas com os números dos artigos publicados, as revistas científicas por tema, principais autores e País de vinculação dos artigos publicados em inglês.

\begin{tabular}{|c|c|c|}
\hline Ano & $\begin{array}{c}\text { Número de artigos } \\
\text { Organizational Sustainability }\end{array}$ & $\begin{array}{c}\text { Número de artigos } \\
\text { Individual Competences }\end{array}$ \\
\hline 2006 & 2 & \\
\hline 2007 & 3 & 3 \\
\hline 2008 & 2 & \\
\hline 2009 & 5 & \\
\hline 2010 & 7 & \\
\hline 2011 & 4 & \\
\hline 2012 & 18 & 3 \\
\hline 2013 & 8 & \\
\hline 2014 & 7 & 2 \\
\hline 2015 & 13 & 2 \\
\hline TOTAL & 69 & 10 \\
\hline
\end{tabular}

Fonte: Autoria Própria (2016)

Foram analisados 79 artigos publicados nos dez anos compreendidos na pesquisa, e verificou-se que no tema Organizational Sustainability do ano de 2006 à 2012 houve uma constante crescente na publicação dos artigos na àrea, onde o seu pico de publicação foi o ano de 2012, e depois disso as publicações sobre o tema foram diminuindo, totalizando 69 artigos nos dez anos verificados. O tema Individual Competences se mostra um assunto extremamente novo no meio acadêmico, totalizando 10 artigos nos dez anos verificados, onde o seu pico de publicação foi de 3 artigos nos anos de 2007 e 2012.Nota-se também que há um predomínio do idioma inglês referente aos artigos analisados.

Destaca-se o trabalho de Rubbo et all (2016), que foram autores do artigo com o título"Sustentabilidade organizacional: Uma análise da produção científica da base Scopus no período de 2005 a 2014", publicado na Revista Espacios (2016). Os autores desenvolveram uma análise utilizando a base SCOPUS no período de 2005 a 2014 sobre o tema de sustentabilidade organizacional. No artigo em questão, os autores filtraram 21 artigos, sendo que foi possível notar uma constante crescente na publicação e no ano de 2014 houve o maior pico de publicações, totalizando 7, sendo que também houve o predomínio do idioma original inglês, e um artigo em português.

O tema de competências individuais não pode ser comparado, pois os autores do artigo não realizaram a pesquisa bibliométrica referente a essa palavra-chave. 
Tabela 2: Revistas Científicas/Base

\begin{tabular}{c|c}
\hline Revistas & Quantidade de artigos Organizational Sustainability \\
\hline Science Direct & 7 \\
\hline Springer & 5 \\
\hline The Learning Organization & 5 \\
\hline Global Business and Organizational Excellence & 4 \\
\hline Greenleaf Publishing & 4 \\
\hline Business Strategy and the Environment & 3 \\
\hline Journal of Accounting \& Organizational Change & 3 \\
\hline Journal of Cleaner Production & 3 \\
\hline Journal of Organizational Change Management & 3 \\
\hline Sustainability & 3 \\
\hline Australian Journal Of Outdoor Education & 2 \\
\hline Business \& Society & 2 \\
\hline Environmental Quality Management & 2 \\
\hline International Journal of Organizational Analysis & 2 \\
\hline Journal of Strategic Information Systems & 2 \\
\hline Journal of Supply Chain Management & 2 \\
\hline Management of Environmental & 2 \\
\hline Organization \& Environment & 2 \\
\hline Outras revistas com 1 artigo publicado em cada & 14 \\
\hline
\end{tabular}

Fonte: Autoria Própria (2016)

Constatou-se que nos dez anos estudados, a base com mais publicações referente ao assunto de sustentabilidade organizacional foi a Science Direct, totalizando 7 artigos neste período. A título de curiosidade a revista é operada pela editora Elsevier, e foi lançada no ano de 1997. Referente a essa palavra-chave, totalizaram-se cerca de 280 artigos, sendo que 36 desses artigos não abriram pois não estavam disponíveis na plataforma, onde desses 280 artigos foram filtrados 69 para um estudo mais aprofundado. As revistas que tiveram apenas 1 artigo publicado em cada totalizaram 14.

Comparando com o artigo da Revista Espacios de Rubbo et all (2016), apenas o periódico científico Journal of Business Ethics possui mais de uma publicação sobre o tema no período de 10 anos, sendo que da palavra-chave totalizaram 127 artigos e apenas um não abriu devido a indisponibilidade do artigo na base SCOPUS, e desses 127 artigos foram filtrados para apenas 21. No artigo, também constatou-se a dispersão nos termos encontrados, onde foi utilizado outras palavras-chaves para complementar o tema de sustentabilidade voltada para a organização.

Tabela 3:Revistas Científicas

\begin{tabular}{c|c}
\hline Revistas & Quantidade de artigos Individual Competences \\
\hline Computers in Industry & 2 \\
\hline Academic Emergency Medicine & 1 \\
\hline
\end{tabular}

43 Revista Competitividade e Sustentabilidade - ComSus, Paraná, v. 3, n. 2, p. 35-48, Jul /Dez. 2016. 


\section{GÜNTHER PIRES WEISSBÖCK - SILVIO ROBERTO STEFANO}

\begin{tabular}{c|l}
\hline Blackwell Publishing & 1 \\
\hline Chain Store Age & 1 \\
\hline Frontiers in Psychology & 1 \\
\hline Int. J. Production Economics & 1 \\
\hline Learning and Individual Differences & 1 \\
\hline Society for Personality Research & 1 \\
\hline Technological Forecasting \& Social Change & 1 \\
\hline
\end{tabular}

Fonte: Autoria Própria (2016)

Referente a palavra-chave sobre competências individuais, nota-se que a revista Computers in Industry é a que possui mais de uma publicação sobre o tema no período estudado. A título de curiosidade, a revista Computers in Industry também é operada pela editora Elsevier. Referente a essa palavra-chave, totalizaram-se cerca de 36 artigos, sendo que 20 desses artigos não abriram pois não estavam disponíveis na plataforma, onde desses 36 artigos foram filtrados 10 para um estudo mais aprofundado.

No artigo de Rubbo et all (2016) da Revista Espacios não houve o estudo sobre essa palavra-chave, não podendo haver uma comparação das bases em estudo.

Tabela 4: Autores

\begin{tabular}{|c|c|c|c|c|}
\hline Stanley J. Hamstra & Goran D. Putnik & AneesJanee Ali & Elias G. Carayannis & FlevyLasrado \\
\hline Marcia Perry & Tom E. Thomas & Chris North & Tairan Huang & Eric Lamm \\
\hline Antony Paulraj & Brooke Moran & Nick Barter & OlaHenfridsson & JiYeonJeong \\
\hline Jane Barnes & Carla Millar & Suzanne Benn & Paul Shrivastava & Stefan Schaltegger \\
\hline Claire Gillet & Anne M. Stoughton & Kent Walker & Paul Burger & Morela Hernandez \\
\hline Shannon Flumerfelt & Peter A.C. Smith & Thomas Leppelt & Dana M. Cosby & Jasmine Tata \\
\hline Rodrigo Lozano & David Gadenne & Cristiana Parisi & PetruL.Curseu & Charles H. Cho \\
\hline Michel L. Ehrenhard & Gretchen Spreitzer & SudhiSeshadri & Martin Kamprath & M. Guerci \\
\hline Angela van der Heijden & Cynthia Scott & RenateWesselink & PhilippSchreck & Tobias Hahn \\
\hline Robert B. Pojasek & Penny Walker & Huo-Tsan Chang & Jonathan P. Doh & Peter A.C. Smith \\
\hline Arjen E.J. Wals & Martin Müller & Britt M. Meyer & ChuxGervaseIwu & Peter Jack Gallo \\
\hline Gillian Rice & Traci Douglas & RebekkahStuteville & Mark Heuer & \\
\hline Maud Besançon & Elaine F. Cassidy & Eric Nelson & Marc J. Epstein & \\
\hline Xavier Boucher & Gareth Enticott & Linda Chalker-Scott & Jonathan Moizer & \\
\hline M. Bennour & Mark G. Edwards & MairaPetrini & Susan Albers Mohrman & \\
\hline Carol A. Adams & CharmayneCullom & Chris Worley & Philip Mirvis & \\
\hline
\end{tabular}

Referente a quantidade de autores por artigo, nota-se que houve uma pequena variação onde um mesmo autor escreveu mais de um artigo por tema. Os autores que escreveram mais de um artigo sobre o mesmo tema foram Gillian Rice, Marc J. Epstein, Rodrigo Lozano \& Peter A.C Smith.

A autora Maira Petrini foi a única brasileira identificada nos 79 artigos, sendo que ela escreveu o artigo referente a sustentabilidade pela revista Journal of Strategic Information 


\section{COMPETÊNCIAS INDIVIDUAIS VOLTADAS À SUSTENTABILIDADE NAS ORGANIZAÇÕ̃S}

Systems e o título de seu artigo "Managing sustainability with the support of business intelligence: Integrating socio-environmental indicators and organisational context" sendo que a universidade vinculada a sua pesquisa foi a Fundação Getulio Vargas.

A titulo de curiosidade, segundo o currículo lattes da autora, o artigo em questão recebeu em 2010 o prêmio de melhor publicação do ano, sendo que a autora continua com diversos projetos de pesquisa na área de sustentabilidade organizacional.

Comparando com o artigo de Rubbo et all (2016) da Espacios, a quantidade de autores por artigo variou entre um e cinco, sendo que um autor teve quatro artigos (19\%), dois autores em dez artigos (48\%), três autores em quatro artigos (19\%), quatro autores em dois artigos $(10 \%)$ e cinco autores em um artigo (5\%), sendo que os demais tiveram um percentual abaixo de $10 \%$.

O artigo de Rubbo et all (2016) da Revista Espacios complementa que não há uma consolidação de autores na área estudada, mas que há 42 autores que possuem mai do que uma publicação na área, variando de 2 a 5 artigos por autor.

Tabela 5: País de vínculação dos artigos publicados

\begin{tabular}{c|c|c}
\hline \multicolumn{2}{c}{ Tabela 5: País de vínculação dos artigos publicados } \\
\hline País & Número de artigos por país & Percentual correspondente \\
\hline Estados Unidos & 32 & $40,50 \%$ \\
\hline França & 7 & $8,86 \%$ \\
\hline Australia & 7 & $8,86 \%$ \\
\hline Canadá & 5 & $6,32 \%$ \\
\hline Alemanha & 5 & $6,32 \%$ \\
\hline Holanda & 5 & $6,32 \%$ \\
\hline Reino Unido & 1 & $6,32 \%$ \\
\hline Portugal & 1 & $1,26 \%$ \\
\hline Malásia & 1 & $1,26 \%$ \\
\hline Brasil & 1 & $1,26 \%$ \\
\hline Itália & 1 & $1,26 \%$ \\
\hline Africa do Sul & 1 & $1,26 \%$ \\
\hline Romênia & 1 & $1,26 \%$ \\
\hline Suiça & 1 & $1,26 \%$ \\
\hline China & 1 & $1,26 \%$ \\
\hline Singapura & 1 & $1,26 \%$ \\
\hline Dinamarca & 1 & \\
\hline Nova Zelândia & 1 & \\
\hline Universidades Não identificadas & $53 \%$ \\
\hline
\end{tabular}

Fonte: Autoria Própria (2016)

Referente ao país de vinculação dos artigos, notamos que os Estados Unidos tem a maior taxa de publicações referente ao tema de sustentabilidade organizacional e 45 Revista Competitividade e Sustentabilidade - ComSus, Paraná, v. 3, n. 2, p. 35-48, Jul /Dez. 2016. 
competências individuais, verificando que há um predomínio da língua inglesa nos artigos filtrados. Outros países que também tem uma taxa significativa de publicações referente a esses dois temas são: França, Austrália, Canadá, Holanda, Alemanha e Reino Unido, e o restante dos países possuem uma taxa menor. Partindo desta afirmação, as universidades que não foram identificadas e o seu respectivo país de vinculação somam 2,53\%.

As universidades que tiveram mais de um artigo publicado nos Estados Unidos foram a College of Business, Arizona State University e Rice University.

Comparando com o artigo de Rubbo et all (2016) da Espacios, nota-se que somente um dos autores não possuía identificação de vinculação e país, sendo que $25 \%$ foram publicados pelos Estados Unidos da América, seguido por Reino Unido, Canadá e Austrália, verificando que também há o predomínio da língua inglesa. Também nota-se que outros países que publicam sobre o tema de sustentabilidade mas tem uma participação menor são: Alemanha, França, Suécia, China e Japão.

Ainda segundo o trabalho de Rubbo et all (2016), verifica-se que somente três artigos (14\%) possui colaboração internacional e evidencia-se um aumento na colaboração internacional nos artigos entre dois ou três países.

\section{CONSIDERAÇÕES FINAIS}

A sustentabilidade organizacional é relevante, pois "quando buscada pelas diversas formas de organizações existentes (empresas, universidades, ONGs, departamentos políticos, etc.), constitui-se em intentos que primam pela busca de um equilíbrio macro de diversos sistemas sociais" (Jiménez-Herrero, 2000).

O estudo analisou 79 artigos referentes ao tema de sustentabilidade organizacional e competências individuais, podendo ser notado que o tema ganhou relevância com o passar do tempo devido a maior competitividade das organizações e a preocupação da sociedade. Referente a produção científica, verificou-se que os artigos foram predominantes de origem americana, a base com mais publicações referente ao tema de sustentabilidade organizacional foi a Sciente Direct com 7 artigos totalizados e referente ao tema de competências individuais a revista com mais publicações foi a Computers in Industry com 2 artigos totalizados.

Identificou-se a totalidade de 75 autores que publicaram apenas um artigo sobre os temas em questão, e apenas 4 autores publicaram mais de um artigo sobre o tema, demonstrando que não há uma elite produtiva. 


\section{COMPETÊNCIAS INDIVIDUAIS VOLTADAS À SUSTENTABILIDADE NAS ORGANIZAÇÕES}

Os países e suas respectivas universidades que tiveram mais de uma publicação referente ao tema foram os Estados Unidos, França, Austrália, Holanda e Reino Unido, sendo que os Estados Unidos possui a maior soma de autores.

Como limitação deste estudo, entende-se que o portfólio foi construído apenas na base de periódicos da CAPES e recomenda-se para futuros estudos pesquisas em outras bases para analisar de forma mais abrangente as publicações referente às competências individuais e sustentabilidade organizacional.

\section{REFERÊNCIAS}

Bacha, M. De L.; Santos, J.; Schaun, A. (2010) Considerações teóricas sobre o conceito de sustentabilidade, VII Simpósio de excelência em gestão e tecnologia, 2010, http://www.aedb.br/seget/arquivos/artigos10/31_cons\%20teor\%20bacha.pdf acesso em 24/08/2016.

Boucher, X.; Bonjour, E.; Grabot, B. (2007) Formalisation and use of competencies for industrial performance optimisation: A survey, Computers in Industry, v.58(2), 98-117, Saint Erienne.

Douglas, T. (2007). Reporting on the Triple Bottom Line at Cascade Engineering, Human Resources Services, v. 26(3), p. 35-43, Global Business and Organizational Excellence, Michigan.

Dutra, J. S. (2008). Gestão de pessoas. São Paulo: Atlas.

Dyllick, T.; Hockerts, K. (2002). Beyound the business case for corporate sustentability. Business Strategy and the environment, v. 11, p. 130-141. Disponível em http://dx.doi.org/10.1002/bse.323 acesso em 03/08/2016.

Edwards, M. G. (2009). An integrative metatheory for organisational learning and sustainability in turbulent times, The Learning Organization, v.16(3), University of Western Australia, Perth, Australia.

Ferreira, L C. (2005). Sustentabilidade: uma abordagem histórica da sustentabilidade. In: BRASIL. Encontros e Caminhos: Formação de Educadoras(es) Ambientais e Coletivos Educadores. Brasília: Ministério do Meio Ambiente.

Fleury, A.; Fleury, M. T. L. (2001). Estratégias empresariais e formação de competências: um quebra cabeças caleidoscópico da indústria brasileira. Rio de Janeiro: Atlas.

Isenmann, R.; Bey, C.; Welter, M. (2007). Online reporting for sustainability issues.Business StrategyandtheEnvironment, $\quad$ v. 16, p. 487-501. Disponível em http://dx.doi.org/10.1002/bse.597 acesso em 10/08/2016.

Jiménez-Herrero, L. M. (2000). Desarrollo Sostenible: transición hacial aco evolución global. Madrid: Pirámide Ediciones.

Loureiro, S. M.; Pereira, V. L. D. do V.; Junior, W. P. (2016). A sustentabilidade e o desenvolvimento sustentável na educação em engenharia, Gestão Educação e Tecnologia Ambiental, v.20(1), Santa Maria, Universidade Federal de Santa Catarina.

47 Revista Competitividade e Sustentabilidade - ComSus, Paraná, v. 3, n. 2, p. 35-48, Jul /Dez. 2016. 
Munck, L.; Munck, M. M., Souza, R. B.; (2011). Sustentabilidade Organizacional: A proposição de uma framework representativa do agir competente para seu acontecimento, Interinstitucional de Psicologia, v.4, edição especial. Disponível em http://www.anpad.org.br/admin/pdf/ESO2915.pdf acesso em 01/08/2016.

Osorio, L. A. R.; Lobato, M. O.; Castillo, X. A. D. (2005). Debates on sustainable development: towards a holistic view of reality. Environment, Development and Sustainability, 7, p. 501-518.

Piccolotto, C. L.; Vassari, S. A; Dutra, J. S. (2010). Percepção de gestores quanto ao papel da área de recursos humanos na gestão de mudança organizacional. In: DUTRA, J. S. (Org.), Gestão de pessoas: práticas modernas e transformação nas organizações. São Paulo: Atlas.

Resende, E. (2000). O Livro das Competências - Desenvolvimento das Competências: a melhor auto-ajuda para pessoas, organizações e sociedade. Rio de Janeiro: Qualitymark.

Rosa, A. (2007). Rede de governança ambiental na cidade de Curitiba e o papel das tecnologias de informação e comunicação. Dissertação de mestrado. Gestão Urbana. Pontifícia Universidade Católica do Paraná.

Rubbo, P.; Pilatti, L. A.; Telles, L. B.; Bittencourt, J. V. M.; Francisco, A. C. (2016). Sustentabilidade organizacional: Uma análise da produção científica da base Scopus no período de 2005 a 2014, Espacios, v.37(13).

Santos, T. (2007). Organizações da sociedade civil e as construções teóricas contemporâneas acerca da sustentabilidade. Anais VI Conferencia Regional de ISTR Para América Latina Y El Caribe. Salvador.

Stefano, S. R.; Alberton, A. (2015). Competências para sustentabilidade e governança nas cooperativas agroindustriais: Uma proposta de análise. XXXIX Encontro da ANPAD, Belo Horizonte.

Wiek, A.; Withycombe, L.; Redman, C. L. (2011). Key competencies in sustainability: a reference framework for academic program development, Integrated Research System for Sustainability Science, Vol. 6(2), p. 203-218, United Nations University.

\section{NOTAS}

${ }^{1}$ Graduando do curso de Administração na Universidade Estadual do Centro-Oeste do Paraná.Voluntário de Iniciação Científica - Unicentro - E-mail: guiweissbock@,hotmail.com

${ }^{2}$ Pós-Doutor em Administração pela Univali (2014). Possui Doutorado em Administração pela Universidade de São Paulo USP (2008). Professor Associado da Universidade Estadual do Centro-Oeste UNICENTRO. Docente do Mestrado Profissional em Administração da UNICENTRO - PPGADM e do Programa de Pós-Graduação em Propriedade Intelectual e de Transferência de Tecnologia para a Inovação - PROFNIT - Mestrado Profissional. Tem experiência profissional na área de Administração, com ênfase em Estratégia e organizações, Gestão de Pessoas, Competências e Sustentabilidade e Comportamento Organizacional. Projetos de Pesquisa financiados pela Fundação Araucária e CNPq. Projeto de Extensão financiado pelo Proext MEC. - E-mail: professor-silvio@hotmail.com 